\title{
Quantum resonance, Anderson localisation and selective rotational excitation in periodically kicked molecules
}

\author{
J. Floß ${ }^{\mathrm{a}}$ and I. Sh. Averbukh \\ Department of Chemical Physics, The Weizmann Institute of Science, 76100 Rehovot, Israel
}

\begin{abstract}
We show that molecules kicked periodically by laser pulses currently used in molecular alignment experiments allow to observe effects of the periodically kicked quantum rotor in a real rotational system. Among these effects are Anderson localisation in angular momentum and the scaling of the quantum resonance. Based on this, we propose a new scheme for selective molecular rotational excitation.
\end{abstract}

\section{Introduction}

The periodically kicked rotor has attracted much attention in the recent decades. In the classical limit the kicked rotor can exhibit truly chaotic motion. However, in the quantum mechanical regime, this chaotic motion is suppressed by Anderson localisation of the wave function in momentum space [1]. On the other hand, when the kicking period is equal to the rotational revival time, the kicked quantum rotor exhibits quantum resonance, an effect which leads to a linear increase of the angular momentum with the number of kicks [2]. It was proposed to observe these phenomena experimentally in linear molecules interacting with microwave pulses [3]. Due to the complexity of generating such pulse trains this proposal has never been realised, and therefore an atom optics analogue of a rotor was used for experimental observations [4].

We propose to use pulse trains of ultra-short optical pulses as used for molecular alignment [5] to observe phenomena of the periodically kicked quantum rotor in a real rotational system. These pulses excite rotational wave packets via Raman-type excitation cascades. It was recently shown that a periodic train of such pulses can induce strong molecular alignment under quantum resonance conditions [6]. We show analytically and numerically that such pulse trains provide a readily available tool to explore the dynamics of the periodically kicked quantum rotor, observing phenomena as the quantum resonance, the scaling of the resonance and Anderson localisation. Also, we show that these effects provide a new toolbox for selective laser manipulations in molecular mixtures.

\section{Model}

We consider interaction of linear molecules with a periodic train of linearly polarised laser pulses. The pulses are far off-resonant from any molecular transition. The interaction potential is given as

$$
V=-\frac{1}{4}\left(\alpha_{\|}-\alpha_{\perp}\right) \cos ^{2} \theta \sum_{n=0}^{N-1} \mathcal{E}^{2}(t-n \tau) .
$$

Here, $\mathcal{E}(t)$ is the temporal envelope of a single laser pulse, $\alpha_{\|}$is the polarisability along the molecular axis and $\alpha_{\perp}$ is the polarisability perpendicular to it, $N$ is the number of pulses, $\tau$ is the period of the

\footnotetext{
a e-mail: johannes.floss@weizmann.ac.il
}

This is an Open Access article distributed under the terms of the Creative Commons Attribution License 2.0, which permits unrestricted use, distribution, and reproduction in any medium, provided the original work is properly cited. 

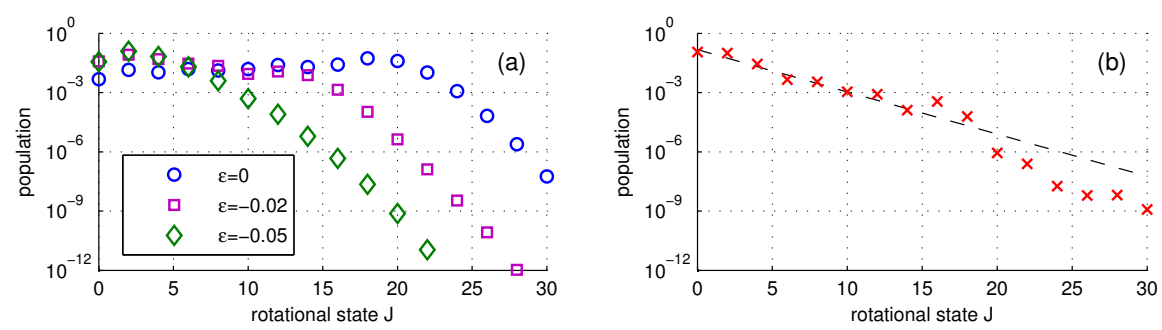

Fig. 1. Population of the rotational levels of ${ }^{15} \mathrm{~N}_{2}$ at $T=10 \mathrm{~K}$ after interaction with a train of eight pulses with $P=3$, for (a) resonant $(\epsilon=0)$ and near-resonant $(|\epsilon| \ll 1)$ kicking, and (b) off-resonant kicking $(\epsilon=-0.44)$. The dashed line indicates the exponential decay caused by Anderson localisation. Due to the selection rules of the Raman type excitation $(\Delta J=0, \pm 2)$, the sub-sets of even and odd rotational levels evolve independently, so for clarity we show only the even ones.

train, and $\theta$ is the angle between the laser polarisation axis and the molecular axis. The pulses are much shorter than the molecular rotational time-scale and therefore they act as $\delta$-kicks. The strength of the interaction can be quantified by $P=\left(\alpha_{\|}-\alpha_{\perp}\right) /(4 \hbar) \int \mathcal{E}^{2}(t) \mathrm{d} t$, which reflects the typical change of the molecular angular momentum (in units of $\hbar$ ) induced by the laser pulse. We measure the energy in units of $2 B$ and the time in units of $\hbar /(2 B)$, where $B$ is the rotational constant. In our simulations we account for thermal effects by averaging over initial states.

\section{Results}

The interaction with the laser pulse creates a rotational wave packet which revives after $t_{r e v}=2 \pi$. A molecule interacting with a pulse train with the period $\tau=t_{\text {rev }}$ experiences strong excitation; this effect is called quantum resonance. We describe the dynamics in close proximity of the quantum resonance, i.e. $\tau=2 \pi+\epsilon$, where $|\epsilon|$ is small, adopting an approach used previously for obtaining a scaling law for the resonances of cold atoms in a pulsed optical standing wave [7]. The exact quantum mechanical dynamics is described by the one-period evolution operator,

$$
\hat{U}=\exp \left(i P \cos ^{2} \theta\right) \exp \left(-i \epsilon \hat{J}^{2} / 2\right),
$$

where $\hat{J}$ is the angular momentum operator. If we rescale the interaction strength to $\tilde{P}=|\epsilon| P$ and the angular momentum to $\tilde{J}=|\epsilon| J, \epsilon$ takes over the role of an effective Planck's constant in Eq. (2) and the dynamics can be described classically. If we further rescale the angular momentum to $I=\tilde{J} /(|\epsilon| P)$ and the time to $x=\sqrt{|\epsilon| P} N$, the classical dynamics depends only on the single scaling variable $x$ (as well as the sign of $\epsilon$ as an parameter). We solve for $I(x)$, average over initial conditions, and divide by the energy for resonant excitation, $E_{\text {res }}=4 P^{2} N^{2} / 15$. The scaled absorbed energy $R(x)$ is found to be

$$
R(x)=\frac{E(|\epsilon|, P, N)}{E_{\text {res }}}=\frac{15}{8} \frac{\left\langle I^{2}(x)\right\rangle}{x^{2}} .
$$

In Figure 1 (a) we show the population of the rotational levels $J$ after a train of eight pulses, for small detuning. Under the condition of exact resonance, the distribution is divided into a flat (in logarithmic scale) plateau region, and a fast decay after some cut-off value of $J$. The cut-off marks the maximum angular momentum supplied by $N$ pulses to a classical rotor (for a rigid rotor this is $J=N P)$, and the fast decay is due to the "tunnelling" into the classically forbidden region. When the detuning is increased, one can see a monotonous deformation of the population curve, whilst the general shape (plateau and fast decay after a cut-off) remains intact.

For larger detuning - shown in Figure 1 (b) -, the distribution is completely different and reflects Anderson localisation in the system. Instead of the plateau, a clear linear (in logarithmic scale) decay over several orders of magnitude is seen, starting from $J=0$. We have verified by additional simulations that this part of the distribution is quite insensitive to variations of the detuning, in contrast to the 


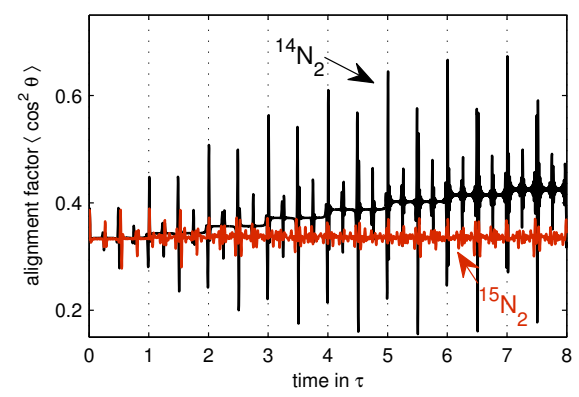

Fig. 2. Alignment factors of ${ }^{14} \mathrm{~N}_{2}$ and ${ }^{15} \mathrm{~N}_{2}$ molecules at $T=298 \mathrm{~K}$, interacting with a train of laser pulses. The pulses are applied with a constant time-delay $\tau$, which is equal to the rotational revival time of ${ }^{14} \mathrm{~N}_{2}(8.38 \mathrm{ps})$.

case of near-resonance kicks. This is an intrinsic property of Anderson localisation in the periodically kicked rotor [1]. The deviations from linearity for $J \gtrsim 20$ are mainly due to the finite number of pulses.

The dependence of the rotational excitation on the detuning $\epsilon$ can be used for isotopologue selective rotational excitation, as reflected in transient beats of molecular alignment. The alignment is quantified by the expectation value of $\cos ^{2} \theta$ (alignment factor). As an example, we consider the two nitrogen isotopologues ${ }^{14} \mathrm{~N}_{2}$ and ${ }^{15} \mathrm{~N}_{2}$, with the rotational revival times $8.38 \mathrm{ps}$ and $8.98 \mathrm{ps}$, respectively. If the pulse train is resonant to ${ }^{14} \mathrm{~N}_{2}$, the detuning for ${ }^{15} \mathrm{~N}_{2}$ is $\epsilon=-0.44$, which corresponds to the far off-resonant case presented in Figure 1 (b). In Figure 2 we show the alignment signal for the two isotopologues interacting with a train of eight pulses. For the resonantly kicked ${ }^{14} \mathrm{~N}_{2}$, strong alignment builds up, in agreement with [6]. On the other hand, for ${ }^{15} \mathrm{~N}_{2}$ the excitation is suppressed by Anderson localisation and the alignment signal remains close to its thermal value.

\section{Conclusion}

Summarising, we have shown that the current laser technology used for non-adiabatic field-free molecular alignment via impulsive Raman-type rotational excitation is sufficient for observing several fundamental phenomena in the dynamics of the periodically kicked quantum rotor [8]. We demonstrated that with such a set-up one can observe the scaling of the quantum resonance and Anderson localisation in a real rotor, a diatomic molecule. Until now, these effects were experimentally observed only in the atom optics substitute of a rotor [4]. We applied the discussed phenomena to the challenging task of selective rotational excitation in a molecular mixture. A new scheme for isotopologue selective excitation, which is based on Anderson localisation in periodically kicked systems, is proposed. Recently, a first experiment following this scheme was done [9].

\section{References}

1. S. Fishman, D. R. Grempel, R. E. Prange, Phys. Rev. Lett. 49, 509 (1982)

2. F. M. Izrailev, D. L. Shepelyanskii, Theor. Math. Phys. 43, 553 (1980)

3. R. Blümel, S. Fishman, U. Smilansky, J. Chem. Phys. 84, 2604 (1986)

4. F. L. Moore, J. C. Robinson, C. Bharucha, B. Sundaraman, M. G. Raizen, Phys. Rev. Lett. 75, 4598 (1995)

5. S. Stapelfeldt, T. Seideman, Rev. Mod. Phys. 75, 543 (2003)

6. J. P. Cryan, P. H. Bucksbaum, R. N. Coffee, Phys. Rev. A 80, 063412 (2009)

7. S.Wimberger, I. Guarneri, S. Fishman, Nonlinearity 16, 1381 (2003)

8. J. Floß, I. Sh. Averbukh, arXiv:1110.3509 (2011)

9. S. Zhdanovich, C. Bloomquist, J. Floß, I. Sh. Averbukh, J. W. Hepburn, V. Milner, Phys. Rev. Lett. (in press, arXiv:1201.3151) 